\title{
Motility Analysis of Pancreatic Adenocarcinoma Cells Reveals a Role for the Atypical $\zeta$ Isoform of Protein Kinase C in Cancer Cell Movement
}

\author{
Carlo Laudanna, Claudio Sorio, Cristina Tecchio, Eugene C. Butcher, \\ Antonio Bonora, Claudio Bassi, and Aldo Scarpa
} Departments of Pathology (CL, CS, CT, AS) and Surgery (AB, CB), University of Verona, Italy; Department of
Pathology (ECB), Stanford University School of Medicine, Stanford, and the Center for Molecular Biology and
Medicine (ECB), the Veteran Affairs Health Care System, Palo Alto, California

\begin{abstract}
SUMMARY: The acquisition of an invasive and metastatic phenotype is accompanied by profound alterations of intracellular mechanisms controlling cell movement. Analysis of quantitative parameters of cell motility in cancer cells may help in the identification of intracellular signaling events determining invasion and metastasis. Here we developed a novel procedure of quantification of cell motility based on time-lapse video microscopy and digital image analysis. Three kinetic parameters, including area change, plasma membrane remodeling, and speed of linear movement, are quantified and combined in one single, time-normalized value we defined motility score (MS). Through calculation of the MS for various human pancreatic adenocarcinoma cell subclones, we identified clones characterized by low or high spontaneous motility in vitro. Analysis of the signaling mechanisms involved in the regulation of pancreatic adenocarcinoma cell motility showed that the atypical $\zeta$ isozyme of the serine-threonine protein kinase C (PKC) plays a critical role in maintaining a high MS in motile subclones, as demonstrated by the inhibitory effect of cell permeable peptides with sequence corresponding to the pseudosubstrate inhibitory region of the atypical $\zeta$ PKC. Other PKC isozymes, either classic or novel, seem not involved. Furthermore, biochemical analysis showed that in motile cells, $\zeta$ PKC is constitutively associated with the plasma membrane, whereas in nonmotile cells, $\zeta$ PKC is totally excluded from the plasma membrane. These data suggest that the disregulation of the function of atypical $\zeta$ PKC might be involved in the acquisition of an invasive and metastatic phenotype in pancreatic adenocarcinoma cells. (Lab Invest 2003, 83:1155-1163).
\end{abstract}

\begin{abstract}
Dancreatic adenocarcinoma is one of the most 1 lethal malignancies, with a 5-year survival rate of $<5 \%$. Surgical removal is only possible in about $15 \%$ of cases, and adjuvant therapies are largely ineffective. Understanding the molecular bases controlling the strong invasive and metastatic attitude of pancreatic cancer could greatly help to develop innovative therapies. Normal epithelial cells establish stable contacts with both adjacent cells and the extracellular matrix, thus maintaining a stable polarized state, which is somehow maintained in preinvasive neoplastic lesions (Hruban et al, 2001). Progression of malignancy induces a switch in cells from a stable adherent to a motile and invasive phenotype.
\end{abstract}

\footnotetext{
DOI: 10.1097/01.LAB.0000081390.92179.F3

Received March 21, 2003.

This work was supported by Fondazione Cassa di Risparmio di Verona (Bando 2001 "Ambiente e Sviluppo Sostenibile"); Ministero Università (Cofin MM06158571 - 2001068593); University of Verona; Istituto Superiore di Sanità (progetto Sclerosi Multipla); Ministero Sanitá (Ricerca ICSO60.2/RFO0-57); and Associazione Italiana per la Ricerca sul Cancro. Address reprint requests to: Dr. Carlo Laudanna, Department of Pathology, University of Verona, Strada Le Grazie 8, 37134 Verona, Italy. E-mail: carlo.laudanna@univr.it
}

Little is known about the motile attitude of pancreatic adenocarcinoma cells and the signaling events controlling their motility. Recent advances in our understanding of the mechanisms involved in cellular motility showed that cell movement comprises a variety of different phenomena. These include cell spreading and polarization, as well as generation of focal adhesion, focal contacts, filopodia, lamellipodia, ruffles, and intercellular junctions. Each of these events is under the control of specialized and distinct signaling pathways, which are likely to be altered in cancer cells (Sahai and Christopher, 2002). In addition many of the signaling molecules controlling cell motility are also involved in the regulation of cell cycle and cell transformation (Olson et al, 1995, 1998; Qu et al, 1995).

The capability of a cancer cell to invade tissues is likely to be associated with the alteration of different aspects of cell motility. Therefore, a purely qualitative analysis of cell motile behavior does not allow for the evaluation of differences in kinetic aspects of cell motility, such as speed of movement, area of spreading, or percentage of plasma membrane remodeled to generate lamellipodia. These aspects are differently under the control of distinct signaling pathways and individually contribute to the overall invasive attitude of cancer cells. The development of methods for a quantitative analysis of cell motility in cancer cells is, 
thus, instrumental to highlight specific modifications of motile behavior correlating them to specific alterations of intracellular signal transduction pathways.

In the present report, we describe a procedure of quantitative calculation of in vitro cancer cell motility, based on time-lapse video microscopy and digital frame-by-frame analysis. We first observed various degrees of spontaneous cell motility in primary cultures of pancreatic adenocarcinoma cells, which led us to establish the novel procedure of motility analysis. In this context we evaluated the role of various isotypes of the serine-threonine protein kinase $\mathrm{C}$ (PKC) in controlling spontaneous high motility of Suit-2 pancreatic cancer cell line. We provide evidence that the atypical $\zeta$ isoform of PKC, but not other PKC isotypes, plays a selective regulatory role in maintaining a highly motile phenotype in pancreatic adenocarcinoma cells.

\section{Results}

\section{Identification of Spontaneous Motile Behavior in Pancreatic Adenocarcinoma Cells and Development of a Quantitative Motility Score (MS)}

Cell motility consists of movement in threedimensional space leading to microenvironmental repositioning. Components of motile behavior, such as cell spreading, generation of filopodia, lamellipodia, and ruffles are clearly identifiable at morphologic analysis. Filopodia, lamellipodia, and ruffles have been morphologically identified according to other investigators (Nobes and Hall, 1995; Ridley et al, 1992). We first looked for evidence of spontaneous cell motility in primary cancer cells. Time-lapse recording of cells from short term cultures of 12 pancreatic cancers allowed for the identification of presence or absence of different motility behaviors. Cancer cells from the four less common pancreatic tumor types, represented by one each of acinar carcinoma, cystadenocarcinoma, intraductal papillary-mucinous carcinoma, and neuroendocrine carcinoma, showed absent or very low spontaneous motility. These cells lacked filopodia, lamellipodia, and ruffles and showed no rearrangement of the occupied area. Conversely, the cancer cells from all eight of the ductal adenocarcinomas showed evidence and variable degrees of motile attitude, consisting in generation of filopodia or lamellipodia and ruffles with a variable degree of rearrangement of the occupied area, accompanied sometimes by the presence of random movement.

This simple morphologic evaluation allowed a rough determination of cancer cell motility. However, the capability to generate filopodia or ruffles may not automatically imply invasive and metastatic capabilities, which also require enhanced linear or random movement and, thus, an alteration of the adhesion-de adhesion turnover mechanism by involving a rapid on/off cycling in integrin activation state. In contrast the occupied area (spreading) does not automatically imply alteration of cytoskeleton remodeling, which is more related to dynamic change of spreading and cell shape over time. Thus, although microscopic observation is sufficient to identify motility in cultured cells, an accurate description of cell motility should be based on a global quantification of the motility-related cellular events by measuring the change of occupied area, the entity of cell surface rearrangement, and the speed of linear movement, possibly combined in one single quantitative parameter.

Based on the above considerations, we used the Suit-2 pancreatic adenocarcinoma cell line as a model system to develop a novel quantitative analysis of cell motility by using time-lapse video microscopy associated to digital frame by frame video analysis. This was based on quantification of three separate aspects of cell motility: area change (AC), surface rearrangement (SR), and speed of movement (M). These three parameters, normalized to the same time frame (one minute), were combined in one single parameter, defined motility score (MS). The AC was expressed as variation coefficient over mean area per time unit (squaredmillimeter per minute). We analyzed single frames, separated by a time grid of 30 seconds, to calculate in squared-micrometers the area of a cell at single time points. The calculated values were then averaged to calculate the mean area over time. This value was normalized to a 1-minute time frame. The standard deviation resulted from the average calculation represents the variation of the area over time, which corresponds, de facto, to a dynamic measurement of area change. To include in the calculation the intrinsic differences in cell volume and shape characteristic of a particular cell, further normalization was performed by calculating the percentage of standard deviation over the mean area. The resulting value, corresponding to the statistic value variation coefficient, was finally considered dynamic measurement of AC over time.

The SR was expressed as percentage of cell perimeter (in micrometers) undergoing filopodia and/or lamellipodia and/or ruffling generation over time (percentage per minute). Single frames, separated by a time grid of 30 seconds, were analyzed to calculate in micrometers the perimeter of a cell at a single time point. Contemporarily, the perimeter undergoing SR was measured. The values were then averaged to calculate the mean values over time. The percentage of cell perimeter regions undergoing surface changes was calculated, normalized to a 1-minute time frame, and considered as the measurement of cell SR.

$M$ was expressed as repositioning in the field over time by using Cartesian coordinates. Single frames, separated by a time grid of 30 seconds, were analyzed to calculate the distance (in micrometers) traveled by a cell over a period of time. The geometrical center of the cell was calculated at each time point, and its position relative to the Cartesian coordinate system of the field was calculated to measure the traveled distance over time. The value was then normalized to 1 minute, expressed as distance over time and considered a measurement of $\mathrm{M}$. 


\section{MS Allows Quantitative Discrimination of Cells with Different Motile Behavior}

The capability of the MS to quantify cell motility was first tested in the context of normal quiescent fibroblasts (Swiss 3T3), as representative of low motility cell, and of human polymorphonuclear (PMN) cells undergoing chemotaxis, as representative of very highly motile cells. Quiescent fibroblasts rearranged, in 1 minute, no more than $1 \%$ of the area occupied by the cell body $(A C=1)$; moreover, no more than $5 \%$ per minute of plasma membrane was remodeled (SR $=5)$, and spatial repositioning was absent $(\mathrm{M}=0)$. Overall, the average MS for normal quiescent fibroblasts was 6 . In contrast, during chemotaxis toward the chemoattractant formyl-Met-Leu-Phe (fMLP), human PMN cells rearranged, in 1 minute, about $50 \%$ of the area occupied by the cell body $(A C=50)$, with $100 \%$ of plasma membrane remodeled $(S R=100)$, and moved at a speed of $15 \mu \mathrm{m} / \mathrm{min}(M=15)$. Overall, the average MS for a PMN cell was 165. Thus, our procedure allows effective quantitative discrimination between cells either characterized by low or very high motile attitude.

\section{Isolation of SUIT-2 Subclones with Different Motile Attitudes}

The Suit-2 pancreatic adenocarcinoma cell line, which we used to develop our procedure of analysis, consisted of a mixed cellular population consisting of both rounded and irregularly shaped cells, the latter suggestive of a spontaneous motile behavior (Fig. 1A). Time-lapse analysis of the original population confirmed the presence of highly motile cells generating lamellipodia, filopodia, and ruffling, as well as cells not showing any motile activity, with a completely round shape and without any SR. This heterogeneity could depend on either different cell cycle stages or presence of different motile and nonmotile subclones. To address this point, the original population was subcloned to obtain monoclonal populations. Sixteen different subclones were isolated, consisting in homogeneous populations of either nonmotile cells or highly motile cells. The former were characterized by round shape and absence of any spontaneous generation of filopodia, lamellipodia, or ruffling (Fig. 1, B and C; clone 1); the highly motile cells showed spontaneous $\mathrm{SR}$, extensive lamellipodia, and ruffling generation (Fig. 1, D and E; clone 4). Importantly, the rate of proliferation was unaffected by the subcloning procedure (data not shown), whereas it led to clones with uniform population of cells showing either $100 \%$ motile or completely nonmotile behavior. Notably, the motile or nonmotile attitude of the isolated clones resulted in a stable phenotype, as it was maintained even after several months of culture and upon liquid $\mathrm{N}_{2}$ storage. Thus, the subcloning procedure allowed effective separation and discrimination among Suit-2derived pancreatic adenocarcinoma cells characterized by opposite motile behavior.
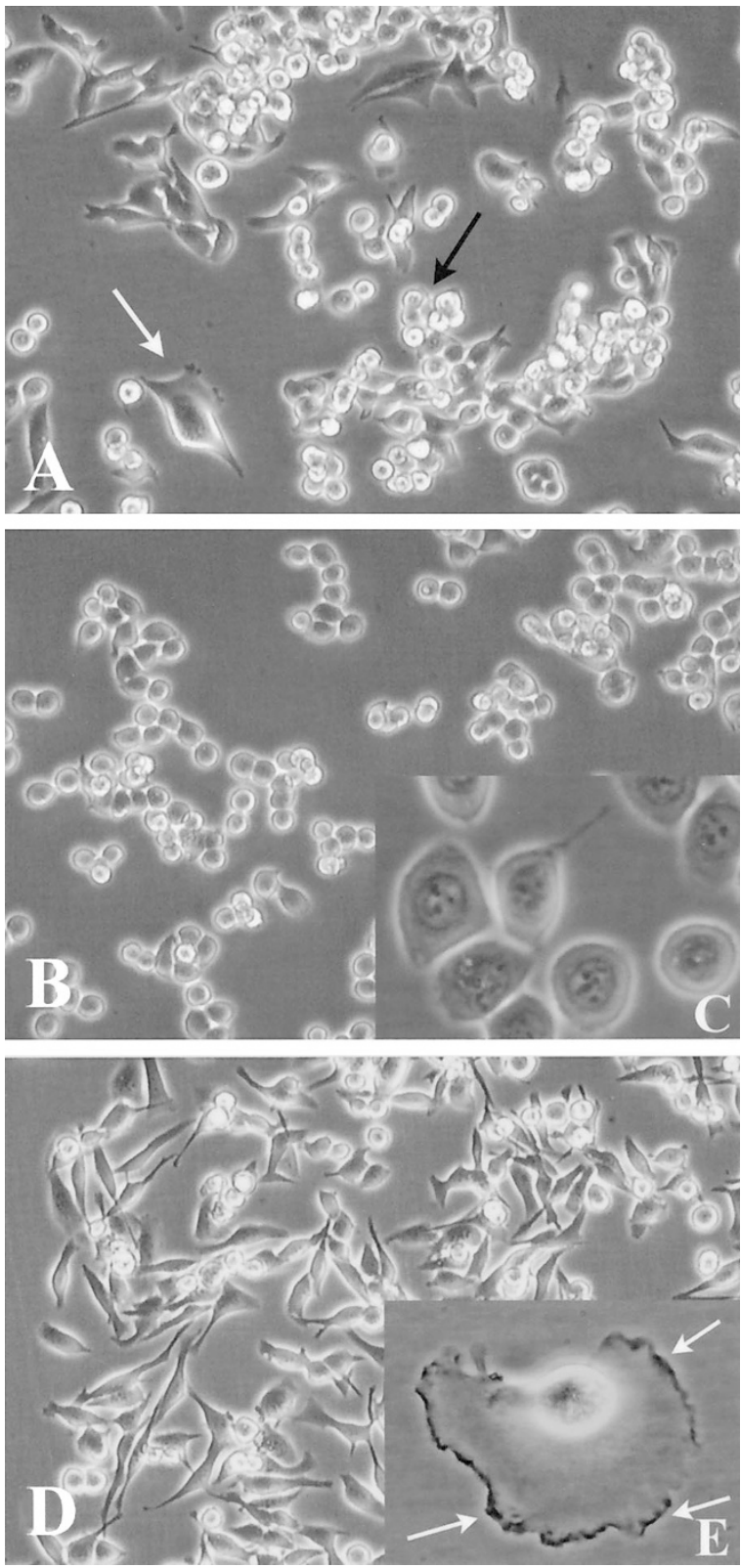

Figure 1.

Subclones of Suit-2 pancreatic adenocarcinoma cells. (A) Original Suit-2 clone showing a mixed population of motile (white arrows) and nonmotile (black arrows) cells. (B and C) Nonmotile subclone 1 ( $\times 20$ magnification, left; $\times 40$ magnification, right); ( $D$ and $E$ ) Motile subclone 4 showing lamellipodia and regions of ruffling indicated by arrows $(\times 20$ magnification, left; $\times 40$ magnification, right). Phase contrast images of cells maintained at $37^{\circ} \mathrm{C}$ in complete Leibovitz's medium.

\section{Quantification of Cell Motility in Pancreatic Adenocarcinoma Cell Subclones}

The analysis of three separate nonmotile clones from Suit-2 cells revealed an MS of 16 (clone 1), 22 (clone 2), and 17 (clone 3). Both the AC and the SR, as well as the $\mathrm{M}$, were rather low (Table 1), suggesting that the oncogenic process in these clones did not affect the intracellular machinery leading to spontaneous cell motility. In contrast, analysis of cells from three separate motile clones revealed an MS of 93 (clone 4), 92 (clone 5), and 88 (clone 6). Thus, the MS of the motile 
Table 1. Quantitative Analysis of Cell Motility in Pancreatic Adenocarcinoma Cell Subclones

\begin{tabular}{lcccc}
\hline & $\begin{array}{c}\text { AC } \\
(\%)\end{array}$ & $\begin{array}{c}\text { SR } \\
(\%)\end{array}$ & $\begin{array}{c}\text { M } \\
(\mu \mathrm{m} / \mathrm{min})\end{array}$ & MS \\
\hline Nonmotile clones & & & & 16 \\
1 & $7 \pm 2$ & $9 \pm 2$ & $2 \pm 1$ & 22 \\
2 & $12 \pm 3$ & $8 \pm 3$ & $1 \pm 0$ & 17 \\
3 & $11 \pm 4$ & $5 \pm 2$ & $4 \pm 2$ & 93 \\
4 & $41 \pm 7$ & $48 \pm 8$ & $2 \pm 0$ & 92 \\
5 & $39 \pm 6$ & $51 \pm 9$ & $4 \pm 1$ & 88 \\
\hline
\end{tabular}

$\mathrm{AC}$, area change; $\mathrm{M}$, speed of movement; MS, motility score; SR, surface rearrangement.

subclones was about 5 times higher than that of nonmotile subclones. Interestingly, the analysis of the MS in the three motile subclones revealed that all three of the score components were altered. Thus, a global and complex alteration of intracellular signaling motor proteins or adhesive receptors is likely to occur in these subclones.

\section{The Role of PKC in Pancreatic Cancer Cell Motility}

The serine-threonine PKC is involved in several motility-related phenomena, including regulation of cytoskeleton polymerization and integrin activation. Thus, we wanted to evaluate the role of PKC family members in pancreatic adenocarcinoma cells motility. Western blot analysis showed that both motile and nonmotile Suit-2 clones expressed the classical $\alpha$ and $\beta$, the novel $\delta$ and $\epsilon$, as well as the atypical $\zeta$ isoforms of PKC (data not shown).

To verify whether PKC is involved in pancreatic cancer cell motility, we first used the PKC inhibitor chelerythrine chloride on the highly motile Suit-2 clone 4. This compound is a specific (but not isozymeselective) PKC inhibitor and represents a unique class of PKC inhibitors that competitively interferes with the phosphate acceptor site and noncompetitively inhibits the ATP binding (Herbert et al, 1990). Chelerythrinechloride treatment almost completely blocked cell motility (Table 2). All of the different components of cell motility were affected, generating a reduction of the MS to 21 from the value of 93 observed in the mock control. This experiment suggests that one or more members of the PKC family are involved in maintaining a spontaneous motile phenotype in pancreatic adenocarcinoma cells.

\section{The $\zeta$ Isoform is the Specific PKC Controlling Pancreatic Cancer Cell Motility}

To further the issue of PKC involvement in cancer cell motility, we took advantage of the capability of synthetic peptides, with sequences identical to the endogenous PKC pseudosubstrate region, to inhibit the activity of the different PKC isoforms. The PKC pseudosubstrate region is an isozyme-selective 17amino acid sequence at the $\mathrm{N}$-terminal of the $\mathrm{C} 1$ domain of the regulatory region of $\mathrm{PKC}$, which mimics the substrate but with a serine-threonine to alanine substitution preventing phosphorylation. In the absence of allosteric activators, this endogenous pseudosubstrate region maintains $\mathrm{PKC}$ in nonactive form. Synthetic peptides derived from this region have been widely used in different cell types as potent and selective inhibitors of PKC phosphorylating activity. The peptides have been synthesized with an $\mathrm{N}$-terminal myristic acid to facilitate their diffusion through the plasma membrane (Laudanna et al, 1998).

Treatment of Suit-2 clone 4 with myristoylated peptides specific for classic $(\alpha)$ and novel ( $\delta$ and $\epsilon$ ) PKCs did not reduce the MS in any of its components (AC, $\mathrm{SR}$, and M) (Table 3). Conversely, the treatment with myristoylated peptides specific to $\zeta$ PKC reduced the

Table 2. Effect of PKC Isotype Inhibition on Pancreatic Adenocarcinoma Cell Motility

\begin{tabular}{|c|c|c|c|c|}
\hline & $\begin{array}{l}\mathrm{AC} \\
(\%)\end{array}$ & $\begin{array}{l}\text { SR } \\
(\%)\end{array}$ & $\begin{array}{c}\mathrm{M} \\
(\mu \mathrm{m} / \mathrm{min})\end{array}$ & MS \\
\hline Mock control & $41 \pm 8$ & $48 \pm 9$ & $4 \pm 1$ & 93 \\
\hline Chelerythrine chloride $5 \mu \mathrm{M}$ & $12 \pm 4$ & $8 \pm 3$ & $1 \pm 0$ & 21 \\
\hline Myr- $\alpha$ PKC $50 \mu \mathrm{M}$ & $39 \pm 7$ & $51 \pm 7$ & $2 \pm 1$ & 92 \\
\hline Myr- $\delta$ PKC $50 \mu \mathrm{M}$ & $37 \pm 8$ & $47 \pm 5$ & $4 \pm 0$ & 88 \\
\hline Myr- $\varepsilon$ PKC $50 \mu \mathrm{M}$ & $47 \pm 6$ & $44 \pm 6$ & $3 \pm 1$ & 94 \\
\hline Myr- $\zeta$ PKC $10 \mu \mathrm{M}$ & $24 \pm 4$ & $35 \pm 8$ & $2 \pm 0$ & 61 \\
\hline Myr- $\zeta$ PKC $25 \mu \mathrm{M}$ & $8 \pm 3$ & $15 \pm 4$ & $1 \pm 0$ & 24 \\
\hline Myr- $\zeta$ PKC $50 \mu \mathrm{M}$ & 0 & $5 \pm 2$ & 0 & 5 \\
\hline Myr- $\zeta$ PKC scr $50 \mu \mathrm{M}$ & $36 \pm 5$ & $51 \pm 8$ & $2 \pm 0$ & 89 \\
\hline
\end{tabular}

$\mathrm{AC}$, area change; M, speed of movement; MS, motility score; SR, surface rearrangement. 
MS in a dose-dependent manner. The highest dose almost completely blocked cell motile behavior in all its components (Table 2). The inhibitory effect of the peptide from $\zeta$ PKC was relatively fast, with an almost complete impairment of cell motility in 30 minutes (Fig. 2). Importantly, cell treatment with the highest dose of a myristoylated peptide with amino acid composition identical to the pseudosubstrate region of the atypical $\zeta$ PKC but with a scrambled sequence did not reduce the MS, thus ruling out a toxic effect due to the myristic acid or a generic peptide-dependent toxic effect. This was further supported by the trypan blue exclusion test and by the capability of Concanavalin A to trigger intracellular calcium movements in cells pretreated with the myristoylated peptide from the pseudosubstrate region of the atypical $\zeta \mathrm{PKC}$ (data not shown).

\section{Intracellular Distribution of $\zeta$ PKC in Motile and Nonmotile Cells}

The above reported data support the role of $\zeta$ PKC in controlling the spontaneous high motility of pancreatic adenocarcinoma cells. Increased $\zeta$ PKC signaling activity has been previously correlated to its enhanced translocation to the light membrane compartments (Laudanna et al, 1998). To further corroborate the role of $\zeta$ PKC in pancreatic adenocarcinoma cell motility, we evaluated its intracellular distribution in motile versus nonmotile cells. Western blot analysis showed that in motile cells $\zeta$ PKC is present in particulate, cytosol, and light membrane fractions. In contrast, in nonmotile cells, although present in particulate and cytosolic fractions, $\zeta$ PKC is totally excluded form the light membrane fraction (Fig. 3, A and B). When we analyzed the same fractions for the presence of the classic isotype $\alpha$ PKC, we found presence of $\alpha$ PKC in all of the three fractions in both motile and nonmotile cells. These data establish a sharp difference between motile and nonmotile subclones, and suggest a correlation between $\zeta$ PKC translocation to the plasma membrane and the acquisition of a motile phenotype in pancreatic adenocarcinoma cells. Furthermore, the

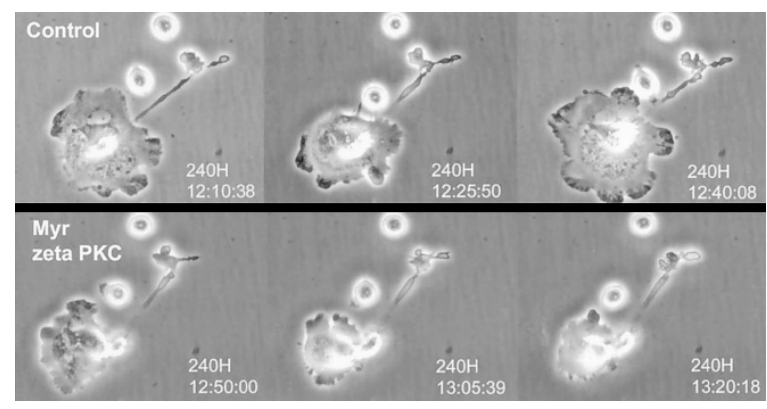

Figure 2.

Effect of myristoylated peptide from the pseudosubstrate region of atypical $\zeta$ protein kinase C (PKC) on motility of subclone 4 from Suit-2 pancreatic adenocarcinoma. Time-lapse frames of cells untreated (control) or treated (Myr- $\zeta$ PKC) with $50 \mu \mathrm{M}$ peptide from $\zeta$ PKC. Phase contrast images of cells maintained at $37^{\circ} \mathrm{C}$ in complete Leibovitz's medium. Shown are images separated by a 15 -minute time frame. Cellular events are accelerated $\times 80$, with respect to real-time.
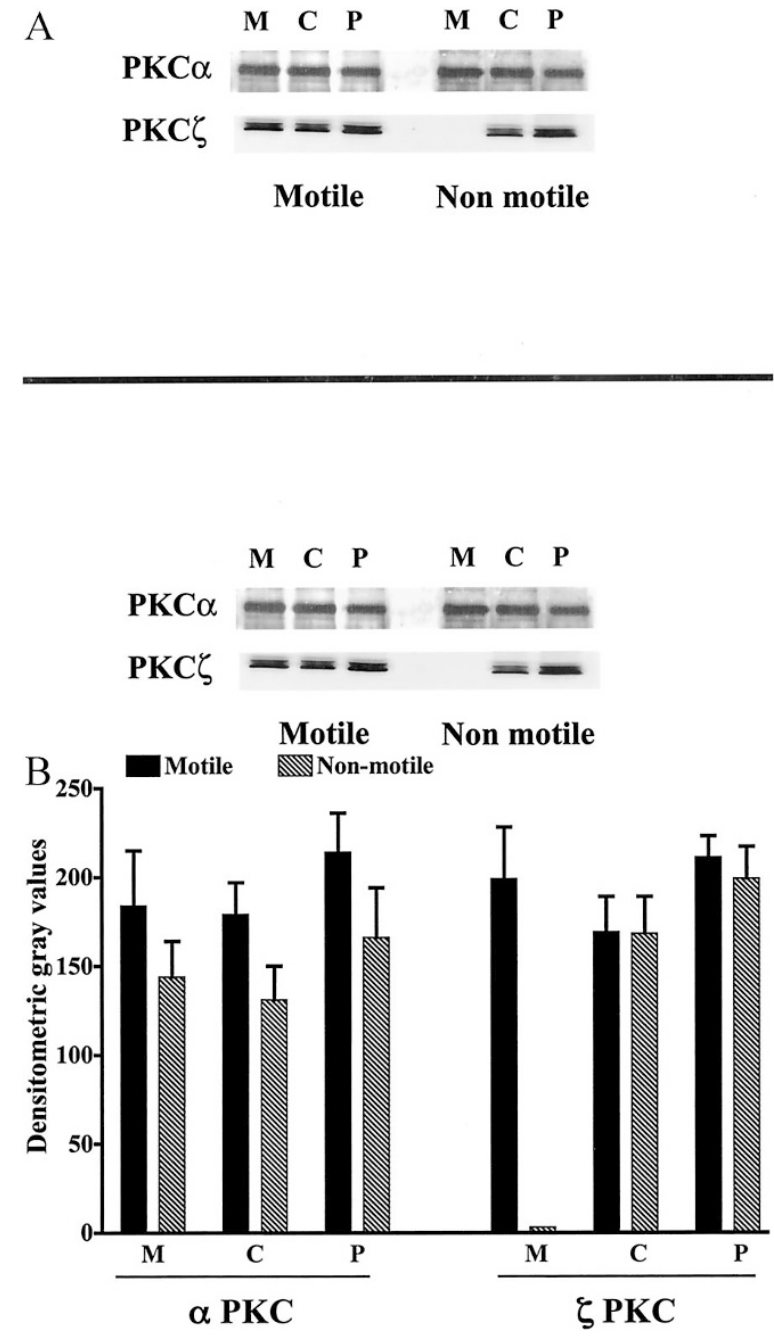

Figure 3.

Intracellular distribution of protein kinase C $(P K C)$ isotypes in motile and nonmotile adenocarcinoma Suit-2 cell clones. (A) Western blot analysis of plasma membrane $(M)$, cytosolic $(C)$ and particulate fractions $(P)$ from motile and nonmotile cell clones, probed with polyclonal antibodies anti- $\zeta$ PKC or $\alpha$ PKC. The blots were probed, after stripping, with both antibodies. (B) Densitometric analysis of Western blots. Plotted numbers are averaged gray level values from three separate experiments presented with sD.

data support the specific role of $\zeta$ PKC versus $\alpha$ PKC in determining differences between motile and nonmotile cells.

\section{Discussion}

The invasive and metastatic capability of malignancies is associated with the acquisition of anomalous motile behavior by cancer cells, in turn dependent of complex physiologic and biochemical cellular changes whose nature is still not clarified. Pancreatic adenocarcinoma is a lethal malignancy whose aggressive behavior depends on several factors, including a strong and early tendency to invasion and metastasis. Even small lesions, less than $1 \mathrm{~cm}$, are associated with invasion of both surrounding tissues and lymphatic vessels.

To investigate mechanisms of cancer cell motility in pancreatic adenocarcinoma, we considered it impor- 
tant to first assess whether cancer cells from human primary pancreatic cancers possessed motile properties. Therefore, we started our studies by investigating the motile attitude of cancer cells in short term primary cultures obtained from fresh samples of surgically removed pancreatic tumors. Time-lapse video microscopy is well suitable to the study of cell motility, and it was essential to our purposes. Among 12 pancreatic cancers we observed, only cells from ductal adenocarcinoma clearly showed variable degrees of spontaneous motility, while cells from each of the four rarer pancreatic cancer types analyzed, including one each of acinar carcinoma, cystadenocarcinoma, intraductal papillary-mucinous carcinoma, and neuroendocrine carcinoma, showed very low to absent motility. This initial evaluation of cell motility, based on observation of the generation of filopodia or lamellipodia and of back movement of lamellipodia on the cell body, also called "ruffling," suggests that the aggressive behavior of ductal adenocarcinoma is seemingly associated with the acquisition of motile properties, which are maintained and observable in in vitro conditions. However, such a purely qualitative observation did neither allow objective comparison among different samples nor was the best experimental procedure to study signaling mechanisms selectively involved in the regulation of different aspects of cancer cell motility.

To overcome these limitations, we developed a quantitative analysis of cell motility allowing the accurate comparison among different tumor samples and correlation between specific phenomena contributing to the overall motile behavior and distinct intracellular signal transduction pathways. This novel method was developed in the context of the pancreatic cancer cell line Suit-2, complemented by a subcloning procedure, which allowed us to separate and identify subclones characterized by sharply different motile behavior. Our approach was instrumental to the analysis of signaling events regulating cell motility in pancreatic adenocarcinoma, which was our main goal.

The results of our study may be summarized as follows: (a) the novel procedure we developed allows one to describe quantitatively the motility attitude of a cell with a single value, the MS; MS is a reproducible quantification that permits direct comparisons among different cell types or samples. Based on quantification of separate aspects of cell motility, MS also allows one to dissect the contribution to the overall normalized value of the different aspects of cell shape remodeling and spatial repositioning; (b) Based on this analysis, we have been able to precisely characterize subclones of the pancreatic ductal cancer cell line Suit-2 showing either low or constitutively high MS; (c) In this experimental setting, specific but non-isozyme selective blockade of serine-threonine PKC blocked cell motility; moreover, isozyme-selective inhibition of the different PKC isoforms by specific myristoylated synthetic blocking peptides showed that $\zeta$ PKC, but no other PKC isozymes, plays a critical role in maintaining a constitutively high MS; and (d) we found a sharp difference in subcellular localization of $\zeta$ PKC (but not PKC) between motile and nonmotile sub- clones, as shown by the constitutive association of $\zeta$ $\mathrm{PKC}$ to the plasma membrane in motile but not in nonmotile cells.

The novel procedure we developed to quantify in vitro motility of cancer cells includes three different parameters, quantified by digital image analysis: AC, $\mathrm{SR}$, and $\mathrm{M}$. These parameters have been chosen with the attempt to include in the analysis as many cellular events indicative of cell movement as possible. In fact, $A C$ is determined by cytoskeleton dynamic and adhesive interaction with the substrate, such as integrin activity. SR mainly involves plasma membrane remodeling, which in turn is regulated by cytoskeleton activity, but is not necessarily dependent on adhesive interactions. $\mathrm{M}$ is also regulated by cytoskeleton activity but also requires rapid on/off states of integrin activity to allow rapid cycling between adhesion and de-adhesion, which is critical to cell microenvironmental repositioning. All three parameters have been normalized with respect to time and combined in one single parameter, the MS, which facilitates data analysis and representation. The MS was clearly able to quantify the difference among nonmotile and very highly motile cells in vitro. In fact, the average MS for normal quiescent fibroblasts was 6, while PMN cells undergoing chemotaxis had an average MS of 165. Application of this novel procedure to the pancreatic ductal cancer line Suit-2 allowed calculation of the MS in different subclones, showing either a low MS (about 20) or a high MS (around 90), and gave then the possibility to analyze signaling mechanisms related to their different motile behavior.

The serine-threonine PKC is involved in the regulation of several aspects of cell adhesion and motility. Particularly, the atypical $\zeta$ isoform of PKC is involved in various aspects of integrin activation and cytoskeleton dynamic interaction (Coghlan et al, 2000; Laudanna et al, 1998). To address the role of PKC in the regulation of the motility of subclones of Suit-2 adenocarcinoma cells, we first used the specific but not isozymeselective PKC inhibitor chelerythrine chloride. This inhibitor completely blocked cell motility, affecting all of the different components of cell motility and generating a reduction of the MS to 21 from the value of 93 observed in the mock control. This data supported a generic role for PKC in motility regulation. Western blot analysis showed that the Suit-2 clones expressed both classic and novel, as well as atypical isoforms of PKC. To investigate the role of different isoforms of PKC, we used myristoylated pseudosubstrate peptides, which display isozyme selective inhibitory capability. Among the different PKC isoforms expressed, only atypical $\zeta-P K C$ isozyme was found to be critical in maintaining a high MS. This data was further supported by the analysis of intracellular distribution of $\zeta$ $\mathrm{PKC}$, showing localization to the plasma membrane in motile cells, whereas in nonmotile cells $\zeta$ PKC is totally excluded from the plasma membrane. This last bit of data is of particular interest, as translocation of PKC to plasma membrane is often required to signaling activity. The constitutive presence of $\zeta$ PKC on plasma membrane in motile Suit-2 subclones supports the 
functional data and suggests that in these cells the biochemical mechanisms supporting $\zeta$-PKC translocation is permanently up-modulated. In contrast, in nonmotile clones these mechanisms are not active. Importantly, in human PMN leukocytes, $\zeta$ PKC is translocated to plasma membrane only upon cell triggering with chemoattractants, and it is mediated by the activation of the small GTPase RhoA (Laudanna et al, 1998). Thus, it is possible that in motile Suit-2 clones, RhoA is permanently active and mediates $\zeta$-PKC localization to the plasma membrane. Interestingly, a role for RhoA and $\mathrm{C}$ in ductal pancreatic adenocarcinoma invasiveness and progression has been previously shown (Kusama et al, 2001; Suwa et al, 1998). Other signaling proteins, such as phosphatidylinositol 3-OH kinase (Gomez et al, 1997; Nakanishi et al, 1993), CDC42 (Coghlan et al, 2000; EtienneManneville and Hall, 2001), and H-ras (Diaz-Meco et al, 1994), are involved in $\zeta-P K C$ regulation. Notably, $\mathrm{H}$-ras is also able to activate the Rho small GTPase cascade (Ridley et al, 1992), and it is a well known direct activator of $\mathrm{PI}(3) \mathrm{K}$ (Rodriguez-Viciana et al, 1994). Thus, H-ras potentially represents a major $\zeta$-PKC regulator. Another ras isoform, K-ras, is found mutated and constitutively active in more than $80 \%$ of pancreatic adenocarcinomas but is wild-type in other pancreatic tumors (Moore et al, 2001a). As the effector regions of $\mathrm{K}$-ras and $\mathrm{H}$-ras (encompassing aa 17-42 and responsible of interaction with downstream effectors) are identical, it is possible that in pancreatic adenocarcinoma cells, mutated K-ras directly regulates $\zeta$-PKC signaling activity. It is important to note, however, that K-ras is mutated also in the nonmotile subclones we analyzed and thus is unlikely to be the only regulator of $\zeta$-PKC subcellular localization. In conclusion, we described a novel method to quantitatively analyze in vitro cancer cell movement. This method allowed us to identify $\zeta$ PKC as a critical intracellular signaling event controlling spontaneous cell motility of ductal pancreatic adenocarcinoma.

\section{Materials and Methods}

\section{Peptides and Other Reagents}

RPMI 1640, fetal bovine serum, glutamine, and Leibovitz's medium were from BioWitthaker, Bergamo, Italy. All myristoylated peptides were synthesized at the Stanford University Protein and Nucleic Acid Facility and stored lyophilized at $4^{\circ} \mathrm{C}$. Peptides were solubilized immediately before use at $1 \mathrm{~mm}$ in PBS, $\mathrm{pH} 7.2$ (Laudanna et al, 1998). The following are peptide sequences from the pseudosubstrate region of human PKC isozymes: $\alpha$-PKC, RFARKGALRQKNVHEVK (positions 19-35); $\delta$-PKC, TMNRRGAIKQAKIHYIK (position 141-157); $\epsilon$-PKC, PAKRQGAVRRRVHQVNG (position 153-169); $\zeta$ PKC, SIYRRGARRWRKLYRAN (positions 113-129); and $\zeta$-PKC scramble, RLRYRNKRIWRSAYAGR. Chelerythrine chloride was from Alexis Biochemicals, (San Diego, California). fMLP and Concanavalin A were from Sigma. Polyclonal antibodies anti-PKC isozymes were from Santa Cruz Biotechnology.

\section{Primary Pancreatic Cancers and Short Term Cultures}

Twelve pancreatic tumor specimens from patients who underwent duodenopancreatectomy were analyzed. These included eight ductal adenocarcinomas and one each of acinar carcinoma, cystadenocarcinoma, intraductal papillary-mucinous carcinoma, and neuroendocrine carcinoma. All patients with ductal adenocarcinomas had either stage-II or -III disease, and none of the 10 patients had either pre- or postadjuvant treatments. After surgical resection, neoplastic tissue was finely minced with scissors and digested for 2 to 4 hours at $37^{\circ} \mathrm{C}$ in collagenase (Biochrom KG, Berlin, Germany). After two washes in PBS, cells were resuspended in RPMI 1640 plus 10\% FCS, 2 mM glutamine, $40 \mu \mathrm{g} / \mathrm{ml}$ gentamicin, and $2 \mu \mathrm{g} / \mathrm{ml}$ amphoterycin $\mathrm{B}$ in a $5 \% \mathrm{CO}_{2}$ atmosphere. After 1 to 2 days, RPMI medium was replaced with Leibovitz L-15 medium, (BioWhittaker) to ensure physiologic $\mathrm{pH}$ in absence of $\mathrm{CO}_{2}$ and spontaneous cell motility in the neoplastic population recorded as described in the "Time-Lapse Video Microscopy" section below.

\section{Cell Lines and Clones}

Swiss 3T3 mouse fibroblasts were form ATCC. The pancreatic adenocarcinoma cell line Suit-2 was originally described by Iwamura et al (1987) and was genetically characterized by Moore et al (2001b). Cells and subclones were maintained in culture in RPMI 1640 plus $10 \%$ FCS and $5 \% \mathrm{CO}_{2}$. Three days before the experiments, the cells were detached, resuspended at low density in RPMI 1640, and plated on six-well plates. Two hours before the experiment, the adherent cells were washed and the RPMI 1640 substituted with Leibovitz's medium containing 10\% FCS.

\section{Time-Lapse Video Microscopy}

For time-lapse analysis of in vitro cell motility, a Panasonic AG-6730 analog S-VHS time-lapse recorder was used. Images were recorded from a highresolution Ikegami CCD video camera connected to an Olympus IX-50 inverted microscope, equipped with phase contrasts optics and an Olympus Peltier thermostated pad to maintain $37^{\circ} \mathrm{C}$. Adherent cells were analyzed at $37^{\circ} \mathrm{C}$ with a $\times 40$ semi-plan apochromatic phase contrast objective. Acquisition of movies was done at a time-frame rate of three frames every 10 seconds (real-time for a PAL system is 25 frame per second). This corresponds to an acquisition of 1125 frame/hr, which leads to a 45-second time-lapse movie. When played and analyzed at a real-time frame rate (25 frame/sec) these parameters of acquisition allow visualization of cell movement at about $\times 80$ real-time speed. Preliminary experiments showed that this was the optimal speed for precise and quantitative evaluation of cell motility.

\section{Digital Analysis and Quantification of Cell Motility}

Time-lapse movies were analyzed by using an Apple computer-based digital image analysis system con- 
sisting of a Scion LG3 PCl frame grabber and of the image analysis software SXM Image by Dr. Steve Barret, United Kingdom (a variation of NIH-Image by Dr. Wayne Rasband, NIH, Bethesda, Maryland). Timelapse movies were digitized to TIFF files, and images were analyzed every 10 frames; this procedure corresponds to a real-time grid analysis of 30 seconds, allowing a maximum of 112 analyzed images from a 45-second movie (60 minutes real-time). Typically, 20 to 40 frames (corresponding to 10 to 20 minutes of motile cell behavior) were analyzed per condition. The different parameters of cell motility were evaluated and quantified by using the SXM image analysis tools. The system was automatically calibrated to transform the number of pixels in micrometers or squaredmicrometers, and a macro file was written to automatically store various measurements of the region of interest to a table for subsequent calculation. Ten to 20 cells for each condition were routinely analyzed.

\section{Isolation of Human PMN Cells and Under Agarose Chemotaxis Assay}

Human blood PMN neutrophils were isolated by dextran sedimentation and centrifugation over FicollHypaque (Pharmacia LKB Biotechnology), as previously described (Laudanna et al, 1998). Contaminating erythrocytes were lysed by hypotonic saline, and neutrophils were washed with PBS and finally resuspended in PBS, $\mathrm{pH} 7.2$, containing $\mathrm{MgCl}_{2} 1 \mathrm{~mm}, \mathrm{CaCl}_{2}$ $1 \mathrm{~mm}$, and $10 \%$ FCS. All of the above procedures were done using reagents prepared in endotoxin-free water for clinical use. Time-lapse acquisition of PMN neutrophils undergoing under agarose chemotaxis toward a $10-n M$ fMLP was done at $37^{\circ} \mathrm{C}$, as previously described (Foxman et al, 1997).

\section{PKC Translocation Assay}

PKC translocation was analyzed as previously described (Laudanna et al, 1998). Briefly, cells were pretreated with diisopropyl-fluoro phosphate $(5.8 \mathrm{~mm}$ for 20 minutes on ice), washed, and then resuspended in ice-cold PBS containing $8 \%$ sucrose, $1 \mathrm{~mm}$ PMSF, and $20 \mu \mathrm{g} / \mathrm{ml}$ leupeptin, pepstatin, aprotinin, and soybean trypsin inhibitor. After scraping off the plates, the cells were sonicated and the homogenates were centrifuged at $800 \times g$ for 10 minutes to remove nuclei and unbroken cells. The postnuclear supernatant was loaded on a discontinuous sucrose gradient (50\% sucrose, $30 \%$ sucrose) and centrifuged for 90 minutes at $100,000 \times \mathrm{g}$. The light membrane fraction (plasma membrane) was collected in the $30 \%$ layer. Following SDS-PAGE on $9 \%$ acrylamide, proteins (10 $\mu \mathrm{g} / \mathrm{lane})$ were electroblotted on $0.2-\mu \mathrm{m}$ pore size nitrocellulose filters, probed with rabbit polyclonal antibodies anti- $\alpha$ or $-\zeta$ PKC (Santa Cruz Biotechnology), followed by goat polyclonal antirabbit HRP conjugated (Sigma) and developed using ECL (Amersham).

\section{References}

Coghlan MP, Chou MM, and Carpenter CL (2000). Atypical protein kinases $\mathrm{Cl}$ and $-\mathrm{z}$ associate with the GTP binding protein Cdc42 and mediate stress fiber loss. Mol Cell Biol 20:2882-2889.

Diaz-Meco MT, Lozano J, Municio M, Berra E, Frutos S, Sanz $\mathrm{L}$, and Moscat $\mathrm{J}$ (1994). Evidence for the in vitro and in vivo interaction of ras with protein kinase $\mathrm{C} \zeta$. J Biol Chem 269:31706-31710.

Etienne-Manneville S and Hall A (2001). Integrin-mediated activation of Cdc42 controls-cell polarity in migrating astrocytes through PKC-zeta. Cell 106:489-498.

Foxman EF, Campbell JJ, and Butcher EC (1997). Multistep navigation and the combinatorial control of leukocyte chemotaxis. J Cell Biol 139:1349-1360.

Gomez J, Garcia A, Borlado LR, Bonay P, Martinez de Aragon A, Silva A, Fresno M, Carrera AC, Eicher-Streiber C, and Rebollo AJ (1997). IL-2 signaling controls actin organization through Rho-like protein family, phosphatidylinositol 3-kinase, and protein kinase C-zeta. J Immunol 158:15161522.

Herbert MJ, Augereu JM, Gleye J, and Maffrand JP (1990). Chelerythrine is a potent and specific inhibitor of protein kinase C. Biochem Biophys Res Commun 172:993-999.

Hruban RH, Adsay NV, Albores-Saavedra J, Compton C, Garrett ES, Goodman SN, Kern SE, Klimstra DS, Kloppel G, Longnecker DS, Luttges J, and Offerhaus GJ (2001). Pancreatic intraepithelial neoplasia: A new nomenclature and classification system for pancreatic duct lesions. Am J Surg Pathol 25:579-586.

Iwamura T, Katsuki T, and Ide K (1987). Establishment and characterization of a human pancreatic cancer cell line (SUIT-2) producing carcinoembryonic antigen and carbohydrate antigen 19-9. Jpn J Cancer Res 78:54-62.

Kusama T, Mukai M, Iwasaki T, Tatsuta M, Matsumoto $\mathrm{Y}$, Akedo $\mathrm{H}$, and Nakamura $\mathrm{H}$ (2001). Inhibition of epidermal growth factor-induced RhoA translocation and invasion of human pancreatic cancer cells by 3-hydroxy-3methyglutaryl-coenzyme A reductase inhibitors. Cancer Res 61:4885-4891.

Laudanna C, Mochly-Rosen D, Liron T, Constantin G, and Butcher EC (1998). Evidence of $z$ protein kinase C involvement in polymorphonuclear neutrophil integrin-dependent adhesion and chemotaxis. J Biol Chem 273:30306-30315.

Moore PS, Orlandini S, Zamboni G, Capelli P, Rigaud G, Falconi M, Bassi C, Lemoine NR, and Scarpa A (2001a). Pancreatic tumors: Molecular pathways implicated in ductal cancer are involved in ampullary but not in exocrine nonductal or endocrine tumorigenesis. Br J Cancer 84:253-262.

Moore PS, Sipos B, Orlandini S, Sorio C, Real FX, Lemoine NR, Gress T, Bassi C, Kloppel G, Kalthoff H, Ungefroren H, Lohr M, and Scarpa A (2001b). Genetic profile of 22 pancreatic carcinoma cell lines: Analysis of K- ras, p53, p16 and DPC4/Smad4. Virchows Arch 439:798-802.

Nakanishi H, Brewer KA, and Exton JH (1993). Activation of the zeta isozyme of protein kinase $C$ by phosphatidylinositol 3, 4, 5-trisphosphate. J Biol Chem 268:13-16.

Nobes CD and Hall A (1995). Rho, rac and CDC42 GTPases regulate the assembly of multimolecular focal complexes associated with actin stress fibers, lamellipodia and filopodia. Cell 81:53-62.

Olson MF, Ashworth A, and Hall A (1995). An essential role for Rho, Rac and CDC42 GTPases in cell cycle progression through $\mathrm{G}_{1}$ Science 269:1270-1272. 
Olson MF, Peterson HG, and Marshall CJ (1998). Signals from Ras and Rho GTPases interact to regulate expression of

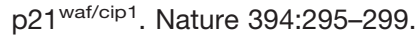

Qiu RG, Ghen J, Kirn D, McCormick F, and Symons M (1995). An essential role for rac in ras transformation. Nature 374 : 457-459.

Ridley A, Paterson HF, Johnston CL, Diekmann D, and Hall A (1992). The small GTP-binding protein rac regulates growth factor-induced membrane ruffling. Cell 70:401-410.

Rodriguez-Viciana $\mathrm{P}$, Warne $\mathrm{PH}$, Dhand R, Vanhaesebroeck B, Gout I, Fry MJ, Waterfield MD, and Downward J (1994). Phosphatidylinositol-3-OH kinase as a direct target of ras. Nature 370:527-532.
Sahai E and Christopher JM (2002). Rho-GTPase and cancer. Nature reviews 2:133-142.

Suwa H, Ohshio G, Imamura T, Watanabe G, Arii S, Imamura M, Narumya S, Hiai H, and Fukumoto M (1998). Overexpression of the rhoC gene correlates with progression of ductal adenocarcinoma of the pancreas. Br J Cancer 77:147-152. 